\title{
Time Reversal Transmission in MIMO Radar
}

\author{
Yuanwei Jin, José M.F. Moura, and Nicholas O’Donoughue \\ Electrical and Computer Engineering \\ Carnegie Mellon University \\ Pittsburgh, PA $15213^{*}$
}

\begin{abstract}
Time reversal explores the rich scattering in a multipath environment to achieve high target detectability. MIMO radar is an emerging active sensing technology that uses diverse waveforms transmitted from widely spaced antennas to achieve increased target sensitivity when compared to standard phased arrays. In this paper, we combine MIMO radar with time reversal to further improve the performance of radar detection. We establish a radar target model in multipath rich environments and develop likelihood ratio tests for the proposed time-reversal MIMO radar (TR-MIMO). Numerical simulations demonstrate improved target detectability compared with the commonly used statistical MIMO strategy.
\end{abstract}

\section{Introduction}

In time reversal (phase conjugation in the frequency domain), a short pulse, for example, transmitted by a source through a dispersive medium, is received by an array, then time reversed, energy normalized, and retransmitted through the same medium. If the scattering channel is reciprocal and sufficiently rich, the retransmitted waveform refocuses on the original source. Our recent work, [1], considers signal detection using time reversal with a pair of transmit/receive antennas. We demonstrate that the time reversal generalized likelihood ratio detector (TR-GLRT) significantly improves detection performance when compared with the conventional detection.

MIMO radar explores multiple-input multiple-output techniques for adaptive transmit and receive beamforming $[2,3]$. The key aspect of a MIMO radar system is the use of a set of orthogonal waveforms simultaneously transmitted from its sparsely placed antennas towards a target area. In a MIMO radar, if the antennas are far enough, the target radar cross sections (RCS) for different transmitting paths become independent random variables. Thus, each orthogonal waveform carries independent information about the target; spatial diversity about the target is thus created. Exploiting the independence between signals at the array elements, a MIMO radar achieves improved detection performance and increased radar sensitivity. This is in contrast with a conventional phased array that presupposes a high correlation between signals either transmitted or received by an array.

${ }^{*}$ This work is funded by the Defence Advanced Research Projects Agency through the Army Research Office under grant no. W911NF-04$1-0031$
Several researchers have developed various models for MIMO radar and provided their interpretation of MIMO radars (see, e.g., $[2,4,5])$. In this paper, we consider a MIMO setup in a rich scattering environment where multipath propagation for targets is sufficient. There are many mechanisms that cause multipath in radar detection, for example, the presence of a large number of scatterers in the vicinity of the target of interest, or tracking and detection of low-angle targets over a flat surface $[6,7]$, etc. Multipath affects the level of the energy return from the target due to coherent combining of the return signals. As a result, we will observe fades and enhancements relative to the level that is expected in a free-space environment. In general, the overall target response is characterized by the target's radar cross section, the multipath propagation due to the surrounding scatterers, and the antenna's aspect angle. The unknown nature of the complex target reflection makes the overall target response appear to be random even for a point target. Therefore, we adopt a statistical model for the target in this paper. Although our MIMO model is somehow similar to what is used in [2], the difference is clear. In [2], the randomness of the target is caused by many reviewing angles from extended targets; in our case, the randomness of a (point) target response is the result of multipath.

We combine time reversal with MIMO radar and develop the binary hypothesis detector. We provide analytical expressions of the test statistics. Time reversal implements matched channel filtering on the transmitter and exploits multipath propagation to its advantage. In previous work $[1,8]$, we showed that time reversal offers higher resolution and improved detectability over conventional methods . In this paper, we demonstrate that a MIMO radar combined with time reversal (TR-MIMO) improves target detectability when compared with statistical MIMO (S-MIMO).

\section{MIMO Modeling}

We consider the problem of detecting a stationary or slowly moving target immersed in a multipath rich scattering environment. Such scenarios occur in many radar applications, for example, detection through tree canopy or low-angle detection and tracking. In this section, we derive the MIMO radar model.

\subsection{Multipath propagation model}

We consider an active multiple antenna system with a pair of stationary antenna arrays A and B. Each antenna array is composed of $N$ omnidirectional antenna elements with uniform inter-element spacing $d$. The two arrays are placed at different locations, which yields a multi-static configuration. Fig. 1 illustrates a two-way radar propagation model 
in multipath. For simplicity, this model considers a twopath propagation with only a single reflected ray emanating from a virtual target image. The two-path propagation is caused by scatterers between the receiving array B and the target. this model can be extended to the more general scenario with multiple path propagation due to scatterers in the fields of view of both the transmitting array A and receiving array B.

The propagation medium between the radar and the target can be characterized by its Green's function, i.e., the channel response at location $\mathbf{r}$ to an impulse at location $\mathbf{r}^{\prime}$. An example of a Green's function is the free space direct path Green's function [9],

$$
G\left(\mathbf{r}, \mathbf{r}^{\prime} ; f\right)=-0.25 j H_{0}^{(2)}\left(f\left|\mathbf{r}-\mathbf{r}^{\prime}\right| / c\right),
$$

where $H_{0}^{(2)}$ is the zeroth-order Hankel function of the second kind, and $c$ is the medium propagation velocity. Here we assume that the medium is reciprocal, i.e., the Green's function satisfies the reciprocity relation: $G\left(\mathbf{r}, \mathbf{r}^{\prime} ; f\right)=$ $G\left(\mathbf{r}^{\prime}, \mathbf{r} ; f\right)$. For a transmit signal $S(f)$, the noise-free received signal at the $n$-th element of array B due to a transmit antenna at array $\mathrm{A}$ is given by

$$
\begin{aligned}
r_{n}(f)= & \xi_{n} e^{-j k \sqrt{X^{2}+\left(Y_{a}-Y_{t}\right)^{2}}}\left(e^{-j k \sqrt{X^{2}+\left(Y_{b}-Y_{t}\right)^{2}}}\right. \\
& \left.+A_{1} e^{-j k \sqrt{X^{2}+\left(Y_{b}+Y_{t}\right)^{2}}}\right) S(f),
\end{aligned}
$$

where: $Y_{a}, Y_{b}$, and $Y_{t}$ are the azimuth coordinates of the antenna at $\mathrm{A}$, the antenna at $\mathrm{B}$, and the target, respectively; $X$ is the target range; $k=\frac{2 \pi}{\lambda}$ is the wavenumber and $\lambda$ is the wavelength at frequency $f$; and $\xi_{n}$ is the complex amplitude due to target characteristics. The complex amplitudes of the direct and reflected rays are simply related by a complex multipath reflection coefficient $A_{1}$. Employing the approximation $\sqrt{1+x} \approx 1+x / 2$ for $x \ll 1$, Eqn. (2) becomes

$$
r_{n}(f)=\eta_{n} e^{-j k \sqrt{X^{2}+\left(Y_{a}-Y_{t}\right)^{2}}} e^{-j k X\left[1+\frac{\left(Y_{b}-Y_{t}\right)^{2}}{X^{2}}\right]} S(f),
$$

where $\eta_{n}=\xi_{n}\left(1+A_{1} e^{-j k \frac{2 Y_{t} Y_{b}}{X}}\right)$ is the combined complex amplitude of the direct and reflected rays. With a large number of scatterers $L$ and $A_{p}, p=1, \cdots, L$, multipath reflection coefficients, the overall target reflectivity is the random variable

$$
\eta_{n}=\xi_{n}\left(1+\sum_{p=1}^{L} A_{p} e^{-j k \frac{2 Y_{t} Y_{b, p}}{X}}\right) .
$$

This analysis implies that, even for a point target, the multipath effect induces fades and enhancements in returned signals relative to the free space returned signals. The transmit and receive antennas provide independent information about the target due to their different viewing angles. We apply the MIMO strategy to this multipath rich environment.

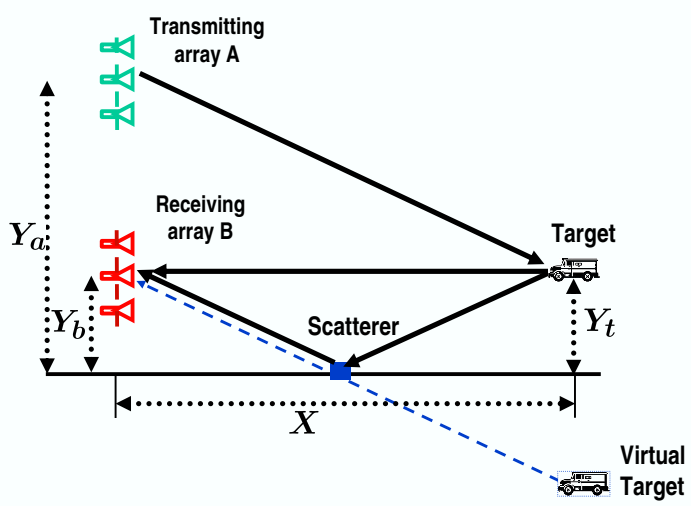

Figure 1. Multipath propagation model.

\subsection{Radar signal model}

We use discrete frequency samples $f_{q}, q=0, \cdots, Q-1$. The number $Q$ is chosen by $Q=\frac{B W}{B_{c}}$, where $B_{c}$ is the coherence bandwidth of the multipath channel, $B W$ is the system bandwidth. The samples taken at frequencies one $B_{c}$ apart are considered to be independent. We let $\mathbf{H}\left(f_{q}\right)$ denote the channel response matrix between the transmit array $\mathrm{A}$ and the receive array $\mathrm{B}$ at $f_{q}$. For simplicity, each array has $N$ antennas. Thus, the $(i, j)$-th entry of $\mathbf{H}\left(f_{q}\right)$, i.e., the channel response from antenna $A_{i}$ to antenna $B_{j}$, can be written as follows:

$$
\left[\mathbf{H}\left(f_{q}\right)\right]_{i, j}=h_{i j}\left(f_{q}\right) \sim \mathcal{C N}\left(0, \sigma_{s}^{2}\right) .
$$

The transmitted signal $s(t)$ is a wideband signal with Fourier representation $S\left(f_{q}\right)$ at angular frequency $f_{q}$. The energy is $E_{s}=\sum_{q=0}^{Q-1}\left|S\left(f_{q}\right)\right|^{2}$. The signal vector received at array B for the $l$-th data snapshot is ${ }^{1}$

$$
\mathbf{y}_{l}\left(f_{q}\right)=\mathbf{H}\left(f_{q}\right) \mathbf{s}_{A}\left(f_{q}\right)+\mathbf{v}_{l}\left(f_{q}\right) .
$$

where $\mathbf{s}_{A}\left(f_{q}\right)$ is the signal vector transmitted from array $\mathbf{A}$

$$
\mathbf{s}_{A}\left(f_{q}\right)=\left[S_{1}\left(f_{q}\right), \cdots, S_{N}\left(f_{q}\right)\right]^{T} .
$$

Conventional detection processes the data received at array $B$. With time reversal, the received radar return at array $\mathrm{B}$ back to array $\mathrm{A}$. So, each data vector $\mathbf{y}_{l}\left(f_{q}\right)$ is time reversed, energy normalized, and retransmitted back into the scattering medium. The $N \times 1$ received signal vector at array $\mathrm{A}$ is

$$
\begin{aligned}
\mathbf{x}_{l}\left(f_{q}\right) & =\mathbf{H}^{T}\left(f_{q}\right)\left[k_{l} \mathbf{y}_{l}\left(f_{q}\right)\right]^{*}+\mathbf{w}_{l}\left(f_{q}\right) \\
& =\mathbf{C}^{*}\left(f_{q}\right) \mathbf{s}_{A}^{*}\left(f_{q}\right)+k_{l} \mathbf{H}^{T}\left(f_{q}\right) \mathbf{v}_{l}^{*}\left(f_{q}\right)+\mathbf{w}_{l}\left(f_{q}\right),
\end{aligned}
$$

where $\mathbf{w}_{l}\left(f_{q}\right) \sim \mathcal{C N}\left(0, \sigma_{n}^{2} \mathbf{I}\right)$ and $\mathbf{v}_{l}\left(f_{q}\right) \sim \mathcal{C N}\left(0, \sigma_{n}^{2} \mathbf{I}\right)$ are independent complex Gaussian noise vectors. The transpose $(\cdot)^{T}$ is used due to the reciprocity condition of the scattering medium

$$
\mathbf{C}\left(f_{q}\right) \triangleq k_{l} \mathbf{H}^{T}\left(f_{q}\right) \mathbf{H}^{*}\left(f_{q}\right)
$$

${ }^{1}$ Here, we assume for the moment that the clutter returns due to dominant surrounding scatterers can be subtracted out. Therefore, only the target response is present. 
where $C_{i, j}\left(f_{q}\right)=k_{l} \sum_{n=1}^{N} h_{n i}\left(f_{q}\right) h_{n j}^{*}\left(f_{q}\right)$ is the $(i, j)$-th entry of $\mathbf{C}\left(f_{q}\right)$. The scalar $k_{l}$ is the energy normalization factor

$$
k_{l}=\sqrt{N E_{s} / \sum_{q}\left\|\mathbf{y}_{l}\left(f_{q}\right)\right\|^{2}} .
$$

For the ideal case where the noise vector contained in the time-reversed signal is $\mathbf{v}\left(f_{q}\right)=0$, we have,

$$
k_{l}=k=\sqrt{N E_{s} / \sum_{q}\left\|\mathbf{H}\left(f_{q}\right) \mathbf{s}_{A}\left(f_{q}\right)\right\|^{2}} .
$$

Such an assumption simplifies the mathematical development. For $\mathbf{v}\left(f_{q}\right) \neq 0$, we rely on numerical means to evaluate the detection performance.

\subsection{Wideband orthogonal waveforms}

Radar systems typically utilize wideband waveforms to achieve fine range resolution. In our problem, the simultaneously transmitted waveforms occupy the same frequency range. To achieve the orthogonality (or quasi-orthogonality) among the transmitted waveforms, we adopt phase coding schemes, [10]. One such design uses complex orthogonal phase vectors $\mathbf{s}_{m}$ by setting its entries to

$$
S_{m}\left(f_{q}\right)=e^{j 2 \pi m q / Q} P\left(f_{q}\right), \quad q=0,1, \cdots, Q-1
$$

for $m=1, \cdots, M \leq Q$ and $P\left(f_{q}\right)=1, \forall q$. For transmit antennas $m$ and $n$, it is straight forward to show that

$$
\sum_{q=0}^{Q-1} S_{m}\left(f_{q}\right) S_{n}^{*}\left(f_{q}\right)=Q \delta(m-n) .
$$

\section{Time Reversal MIMO Detector}

Let $\mathbf{x}_{l}\left(f_{q}\right)=\left[X_{l, 1}\left(f_{q}\right), \cdots, X_{l, N}\left(f_{q}\right)\right]^{T}$ be the collection of $N$ received signals at $f_{q}$ for the $l$-th data snapshot, where

$$
X_{l, i}\left(f_{q}\right)=\sum_{j=1}^{N} C_{i j}\left(f_{q}\right) S_{j}^{*}\left(f_{q}\right)+W_{l, i}\left(f_{q}\right) .
$$

Matched filtering the received signals with the orthogonal waveforms and collecting all the frequency components, we obtain the following signals:

$$
R_{l, i n}=\sum_{q} X_{l, i}\left(f_{q}\right) S_{n}^{*}\left(f_{q}\right)=\widetilde{C}_{i n}+\widetilde{W}_{l, i n},
$$

where

$$
\begin{aligned}
\widetilde{C}_{i n} & =\sum_{q} \sum_{j} C_{i j}\left(f_{q}\right) S_{j}\left(f_{q}\right) S_{n}^{*}\left(f_{q}\right), \\
\widetilde{W}_{l, i n} & =\sum_{q} W_{l, i}\left(f_{q}\right) S_{n}^{*}\left(f_{q}\right) .
\end{aligned}
$$

We notice that $E\left\{C_{i j}\left(f_{q}\right)\right\}$ is frequency independent, hence

$$
\begin{aligned}
E\left\{\widetilde{C}_{i n}\right\} & =\sum_{q} \sum_{j} E\left\{C_{i j}\left(f_{q}\right)\right\} S_{j}\left(f_{q}\right) S_{n}^{*}\left(f_{q}\right) \\
& =\sum_{j} E\left\{C_{i j}\left(f_{q}\right)\right\} Q \delta(n-j) \\
& =Q E\left\{C_{i n}\left(f_{q}\right)\right\}
\end{aligned}
$$

i.e., the transmitting waveforms are quasi-orthogonal. We then use the following approximation:

$$
\widetilde{C}_{i n} \approx \sum_{q=0}^{Q-1} C_{i n}\left(f_{q}\right)
$$

In particular, from the diagonal components $(i=n)$ of the matrix $\mathbf{C}\left(f_{q}\right)$, we obtain the real-valued data

$$
\widetilde{C}_{i i}=k \sum_{q=0}^{Q-1} \sum_{j=1}^{N}\left|h_{i j}\left(f_{q}\right)\right|^{2} .
$$

Eqn. (18) indicates that, by time reversal, the diagonal entries of the time-reversed target channel response are focused and become dominant compared with the (complex valued) off-diagonal entries. This observation yields a simpler detector by considering only the diagonal terms given by (18). Grouping $\left\{R_{l, i i}\right\}$ into $N$ by 1 vectors yields $\mathbf{r}_{l}^{D}=\left[R_{l, 11}, R_{l, 22}, \cdots, R_{l, N N}\right]^{T}, \widetilde{\mathbf{c}}_{l}^{D}=$ $\left[\widetilde{C}_{11}, \cdots, \widetilde{C}_{N N}\right]^{T}, \widetilde{\mathbf{w}}_{l}^{D}=\left[\widetilde{W}_{l, 11}, \cdots, \widetilde{W}_{l, N N}\right]^{T}$. The binary hypothesis test for TR-MIMO using diagonal terms ( $N$ terms) is given by

$$
\begin{aligned}
& \mathbb{H}_{1}: \mathbf{r}_{l}^{D}=\widetilde{\mathbf{c}}_{l}^{D}+\widetilde{\mathbf{w}}_{l}^{D} \\
& \mathbb{H}_{0}: \mathbf{r}_{l}^{D}=\widetilde{\mathbf{w}}_{l}^{D} .
\end{aligned}
$$

Certainly, one can use all the elements of the matrix $\mathbf{C}\left(f_{q}\right)$. In this case, the binary hypothesis test for TR-MIMO using all terms $\left(N^{2}\right.$ terms) is given by

$$
\begin{aligned}
& \mathbb{H}_{1}: \mathbf{r}_{l}^{F}=\widetilde{\mathbf{c}}_{l}^{F}+\widetilde{\mathbf{w}}_{l}^{F} \\
& \mathbb{H}_{0}: \mathbf{r}_{l}^{F}=\widetilde{\mathbf{w}}_{l}^{F}
\end{aligned}
$$

where the $N^{2}$ by 1 data vectors are $\mathbf{r}_{l}^{F}=$ $\left[R_{l, 11}, R_{l, 12}, \cdots, R_{l, N N}\right]^{T}, \widetilde{\mathbf{c}}_{l}^{F}=\left[\widetilde{C}_{11}, \widetilde{C}_{12}, \cdots, \widetilde{C}_{N N}\right]^{T}$, $\widetilde{\mathbf{w}}_{l}^{F}=\left[\widetilde{W}_{l, 11}, \widetilde{C}_{12}, \cdots, \widetilde{W}_{l, N N}\right]^{T}$. Later, in Section 5, we show that the performance difference between these two cases, i.e., (19) and (20), is small. However, the mathematical analysis for (19) is easier. Thus, we provide analytical results for (19) only and examine the performance of (20) by numerical means.

For S-MIMO, we construct the detector similar of the detector given in [2]. Let $\mathbf{y}_{l}\left(f_{q}\right)=\left[Y_{l, 1}\left(f_{q}\right), \cdots, Y_{l, N}\left(f_{q}\right)\right]^{T}$ be the collection of $N$ received signals, where

$$
Y_{l, i}\left(f_{q}\right)=\sum_{j=1}^{N} h_{i j}\left(f_{q}\right) S_{j}\left(f_{q}\right)+V_{l, i}\left(f_{q}\right) .
$$


Matched filtering the received signals by orthogonal waveforms yields

$$
U_{l, i n}=\sum_{q} Y_{l, i}\left(f_{q}\right) S_{n}^{*}\left(f_{q}\right)=\widetilde{h}_{i n}+\widetilde{V}_{l, i n},
$$

where

$$
\begin{aligned}
\widetilde{h}_{i n} & =\sum_{q} \sum_{j} h_{i j}\left(f_{q}\right) S_{j}\left(f_{q}\right) S_{n}^{*}\left(f_{q}\right), \\
\widetilde{V}_{l, i n} & =\sum_{q} V_{l, i}\left(f_{q}\right) S_{n}^{*}\left(f_{q}\right) .
\end{aligned}
$$

We notice that $E\left\{h_{i j}\left(f_{q}\right)\right\}$ is frequency independent, hence

$$
\begin{aligned}
E\left\{\widetilde{h}_{i n}\right\} & =\sum_{q} \sum_{j} E\left\{h_{i j}\left(f_{q}\right)\right\} S_{j}\left(f_{q}\right) S_{n}^{*}\left(f_{q}\right) \\
& =\sum_{j} E\left\{h_{i j}\left(f_{q}\right)\right\} Q \delta(n-j)=Q E\left\{h_{i n}\left(f_{q}\right)\right\}
\end{aligned}
$$

Again, the transmitting waveforms are quasiorthogonal. We use the following approximation: $\widetilde{h}_{i n} \approx \sum_{q=0}^{Q-1} h_{i n}\left(f_{q}\right)$. Grouping $U_{l, i n}$ into $N^{2} \times 1$

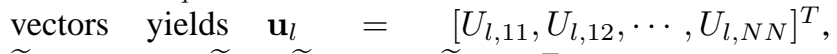
$\widetilde{\mathbf{h}}_{l}=\left[\widetilde{h}_{l, 11}, \widetilde{h}_{l, 12}, \cdots, \widetilde{h}_{l, N N}\right]^{T}, \quad$ and $\widetilde{\mathbf{v}}_{l}=$ $\left[\widetilde{V}_{l, 11}, \widetilde{V}_{l, 12}, \cdots, \widetilde{V}_{l, N N}\right]^{T}$. Thus, the binary hypothesis test for S-MIMO is given by

$$
\begin{aligned}
& \mathbb{H}_{1}: \mathbf{u}_{l}=\widetilde{\mathbf{h}}_{l}+\widetilde{\mathbf{v}}_{l} \\
& \mathbb{H}_{0}: \mathbf{u}_{l}=\widetilde{\mathbf{v}}_{l} .
\end{aligned}
$$

\section{Performance Analysis}

In this section, we derive the test statistics for (19) and (25). A closed form for the probability density function is often difficult to obtain. Thus, we will rely on approximations. In what follows, we use $\chi_{M}^{2}(\mu)$ to represent the Chi-squared distribution with $M$ degrees of freedom and non-centrality parameter $\mu$. In (18), $\left|h_{i j}\left(f_{q}\right)\right|^{2} \sim \frac{\sigma_{s}^{2}}{2} \chi_{2}^{2}$ are independent and identically distributed random variables. The symbol stands for "distributed as." Let's define

$$
Z_{i}\left(f_{q}\right) \triangleq k \sum_{j=1}^{N}\left|h_{i j}\left(f_{q}\right)\right|^{2} \sim \bar{k} \frac{\sigma_{s}^{2}}{2} \chi_{2 N}^{2}
$$

where $\bar{k}=E\{k\}$. The sequence $\left\{Z_{i}\left(f_{q}\right), \forall q\right\}$ is i.i.d, with finite variance. By the central limit theory, the sequence $\left\{Z_{i}\left(f_{q}\right)\right\}$ is asymptotically Gaussian, i.e.,

$$
\frac{1}{\sqrt{Q}} \widetilde{C}_{i i} \sim \mathcal{N}(\mu, \Phi)
$$

where

$$
\begin{aligned}
\mu & =E\left\{Z_{i}\left(f_{q}\right)\right\}=\bar{k} N \sigma_{s}^{2} \\
\Phi & =\operatorname{Var}\left\{Z_{i}\left(f_{q}\right)\right\}=\bar{k}^{2} N \sigma_{s}^{4}
\end{aligned}
$$

This also implies that $\sum_{i=1}^{N} \frac{1}{Q} \widetilde{C}_{i i}^{2} \sim \Phi \chi_{N}^{2}\left(\gamma^{2}\right)$, where the non-centrality parameter is $\gamma^{2}=\sum_{i=1}^{N} \frac{\mu^{2}}{\Phi}=N^{2}$. The binary hypothesis test given in (19) can be written approximately as

$$
\ell_{\mathrm{TR}}=\left\|\mathbf{r}_{l}^{D}\right\|^{2} \sim \begin{cases}Q \Phi \chi_{N}^{2}\left(\gamma^{2}\right)+Q \frac{\sigma_{n}^{2}}{2} \chi_{2 N}^{2} & \mathbb{H}_{1} \\ Q \frac{\sigma_{n}^{2}}{2} \chi_{2 N}^{2} & \mathbb{H}_{0}\end{cases}
$$

Notice that the test statistic under $\mathbb{H}_{1}$ is the weighted sum of a non-central chi-squared random variable and an independent central chi-squared random variable. A closed form for the distribution of the test statistics is difficult to obtain. We wish to approximate it in the form $g \chi_{h}^{2}\left(\xi^{2}\right)$. By modifying Theorem 3.1 in [11], we obtain the following equivalent binary hypothesis test for TR-MIMO given in (30):

$$
\ell_{\mathrm{TR}}^{\prime}=\left\|\mathbf{r}_{l}^{D}\right\|^{2} \sim\left\{\begin{array}{cc}
g \chi_{h}^{2}\left(\xi^{2}\right) & \mathbb{H}_{1} \\
\frac{\sigma_{n}^{2}}{2} \chi_{2 N}^{2} & \mathbb{H}_{0}
\end{array}\right.
$$

where the scaler $g$ and the degrees of freedom $h$ are given by

$$
g=\frac{\sum_{i=1}^{2} \nu_{i} \lambda_{i}^{2}}{\sum_{i=1}^{2} \nu_{i} \lambda_{i}}, \quad h=\frac{\left(\sum_{i=1}^{2} \nu_{i} \lambda_{i}^{2}\right)^{2}}{\sum_{i=1}^{2} \nu_{i} \lambda_{i}^{2}},
$$

where $\xi^{2}=\Phi Q \gamma^{2} / g, \nu_{1}=N, \nu_{2}=2 N, \lambda_{1}=\Phi, \lambda_{2}=$ $\sigma_{n}^{2} / 2$. Next, we examine the detection probability and the false alarm rate.

TR-MIMO Let $\eta$ denote the decision threshold, we have

$$
P_{\mathrm{FA}}=P_{r}\left(\ell_{\mathrm{TR}}>\eta \mid \mathbb{H}_{0}\right)=P_{r}\left(\chi_{2 N}^{2}>\frac{2 \eta}{\sigma_{n}^{2}}\right) .
$$

The threshold for TR-MIMO can be obtained by

$$
\eta_{\mathrm{TR}-\mathrm{MIMO}}=\frac{\sigma_{n}^{2}}{2} \psi_{\chi_{(2 N, 0)}^{2}}^{-1}\left(1-P_{\mathrm{FA}}\right)
$$

where $\psi_{\chi_{(M, \delta)}^{2}}^{-1}(\cdot)$ is the inverse cumulative distribution function of a chi-squared random variable with $M$ degrees of freedom and non-centrality parameter $\delta$. The probability of detection is given by

$$
\begin{aligned}
P_{\mathrm{D}}^{\mathrm{TR}} & =P_{r}\left(\ell_{\mathrm{TR}}>\eta \mid \mathbb{H}_{1}\right)=P_{r}\left(g \chi_{h}^{2}\left(\xi^{2}\right)>\eta\right) \\
& =1-\psi_{\chi_{\left(2 N, \xi^{2}\right)}^{2}}\left(\frac{\eta}{g}\right) \\
& =1-\psi_{\chi_{\left(2 N, \xi^{2}\right)}^{2}}\left(\frac{\sigma_{n}^{2} \psi_{\chi_{(2 N, 0)}^{2}}^{-1}\left(1-P_{\mathrm{FA}}\right)}{2 g}\right)
\end{aligned}
$$

S-MIMO The binary hypothesis test (25) for S-MIMO is given by (using the full $N$ by $N$ channel matrix)

$$
\ell_{\mathrm{S}}=\left\|\mathbf{u}_{l}\right\|^{2} \sim \begin{cases}Q\left(\frac{\sigma_{s}^{2}}{2}+\frac{\sigma_{n}^{2}}{2}\right) \chi_{2 N N}^{2} & \mathbb{H}_{1} \\ Q \frac{\sigma_{n}^{2}}{2} \chi_{2 N N}^{2} & \mathbb{H}_{0}\end{cases}
$$




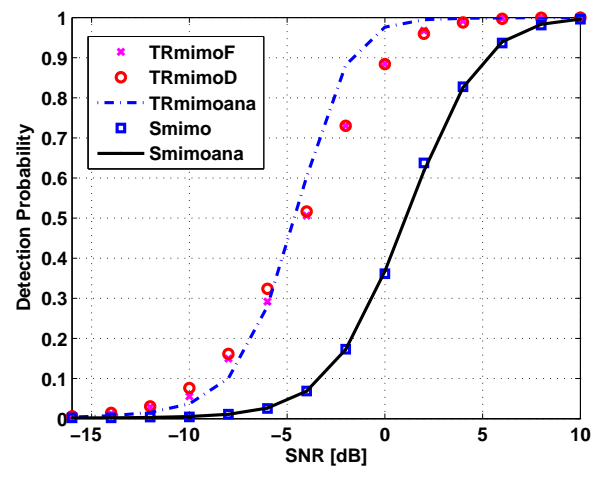

Figure 2. ROC for TR-MIMO vs. S-MIMO

The detection probability is given by

$$
\begin{aligned}
P_{\mathrm{D}}^{\mathrm{S}} & =P_{r}\left(\ell_{\mathrm{S}}>\eta \mid \mathbb{H}_{1}\right)=P_{r}\left(Q \frac{\sigma_{s}^{2}+\sigma_{n}^{2}}{2} \chi_{2 N N}^{2}>\eta\right) \\
& =1-\psi_{\chi_{(2 N N, 0)}^{2}}\left(\frac{2 \eta}{Q\left(\sigma_{s}^{2}+\sigma_{n}^{2}\right)}\right) \\
& =1-\psi_{\chi_{(2 N N, 0)}^{2}}\left(\frac{\sigma_{n}^{2} \psi_{\chi_{(2 N N, 0)}^{2}}^{2}\left(1-P_{\mathrm{FA}}\right)}{\sigma_{s}^{2}+\sigma_{n}^{2}}\right)
\end{aligned}
$$

\section{Numerical Simulations}

In this section, we carry out numerical simulations to evaluate the performance of the proposed detectors. We use two transmit antennas and two receive antennas $(N=2)$. We choose the number of frequencies $Q=4$ for simulation purpose. The signal-to-noise ratio is defined as $\mathrm{SNR}=\sigma_{s}^{2} / \sigma_{n}^{2}$. The false alarm rate is $P_{\mathrm{FA}}=0.001$. Fig. 2 depicts the ROC curve for TR-MIMO vs. S-MIMO for the ideal case where the noise vector in the time-reversed signal is zero. The analytical results are consistent with the Monte Carlo simulation results. The proposed TR-MIMO has about $5 \mathrm{~dB}$ gain over S-MIMO. As anticipated, the TR-MIMO using diagonal terms (19) has almost identical performance to the TR-MIMO using all terms (20). Fig. 3 depicts the ROCs of TR-MIMO vs. S-MIMO where the time-reversed signal is contaminated by noise. In this case, we still observe about $3 \mathrm{~dB}$ gain of TR-MIMO over S-MIMO.

\section{Conclusion}

This paper develops the time reversal MIMO radar detector and provides an approximate closed-form for the probability distribution of TR-MIMO. The algorithm we develop is robust in rich multipath environments and shows a significant gain over the statistical MIMO detector.

\section{References}

[1] J. M. F. Moura and Y. Jin, "Detection by time reversal: single antenna," IEEE Transactions on Signal Processing, vol. 55, no. 1, pp. 187-201, January 2007.

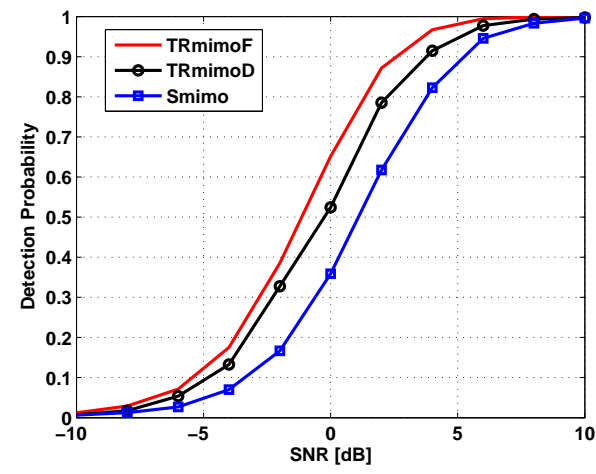

Figure 3. ROC for TR-MIMO vs. S-MIMO

[2] E. Fishler, A. Haimovich, R. Blum, D. Chizhik, L. Cimini, and R. Valenzuela, "MIMO radar: an idea whose time has come," in Proceedings of the IEEE Radar Conference. IEEE, April 2004, pp. 71-78.

[3] G. J. Frazer, Y. I. Abramovich, and B. A. Johnson, "Spatially waveform diverse radar: perspectives for high frequency OTHR," in IEEE Radar Conference. IEEE, April 2007.

[4] D. R. Fuhrmann and G. S. Antonio, "Transmit beamforming for MIMO radar systems using partial signal correlation," in Proceedings of the 38th Asilomar Conference on Signals, Systems, and Computers, vol. 1. Pacific Grove, CA: IEEE, November 2004, pp. 295299.

[5] F. Robey, S. Coutts, D. Weikle, J. McHarg, and K. Cuomo, "MIMO radar theory and experimental results," in Proceedings of the 38th Asilomar Conference on Signals, Systems, and Computers, vol. 1. Pacific Grove, CA: IEEE, November 2004, pp. 300-304.

[6] S. L. Wilson and B. D. Carlson, "Radar detection in multipath," IEE Proceedings - Radar, Sonar Navig., vol. 146, no. 1, pp. 45-54, February 1999.

[7] E. Bosse, R. Turner, and M. Lecours, "Tracking Swerling fluctuating targets at low altitude over the sea," IEEE Transactions on Aerospace and Electronics Systems, vol. 27, no. 5, pp. 806-822, September 1991.

[8] Y. Jin and J. M. F. Moura, "TR-SAR: time reversal target focusing in spotlight SAR," in ICASSP'07, IEEE International Conference on Signal Processing, vol. 2. Honolulu, HI: IEEE, April 2007, pp. 957-960.

[9] P. M. Morse and H. Feshback, Methods of Theoretical Physics, Part I. New York, NY: McGraw-Hill, 1953.

[10] D. R. Wehner, High-Resolution Radar. Boston, MA: Artech House, 1995.

[11] G. E. P. Box, "Some theorems on quadratic forms applied in the study of analysis of variance problems," The Annals of Mathematical Statistics, vol. 25, no. 2, pp. 290-302, June 1954. 